\title{
Les élites européennes comme champ(s)
}

Réflexions sur les usages de la prosopographie et de l'analyse géométrique des données à partir de trois expériences de recherche collective sur des objets transnationaux

European Elites as field(s). Reflections on the uses of prosopography and Geometric Data Analysis based on three collective research experiences on transnational objects

\section{Frédéric Lebaron}

\section{(2) OpenEdition}

\section{Journals}

\section{Édition électronique}

URL : http://journals.openedition.org/conflits/19305

DOI : 10.4000/conflits. 19305

ISSN : 1777-5345

Éditeur :

CCLS - Centre d'études sur les conflits lilberté et sécurité, L'Harmattan

Édition imprimée

Date de publication : 8 août 2016

Pagination : 121-147

ISBN : 978-2-343-10075-3

ISSN : 1157-996X

Référence électronique

Frédéric Lebaron, «Les élites européennes comme champ(s) », Cultures \& Conflits [En ligne], 102 | été 2016, mis en ligne le 08 août 2018, consulté le 30 mars 2021. URL : http://journals.openedition.org/ conflits/19305; DOI : https://doi.org/10.4000/conflits.19305 


\title{
Les élites européennes comme champ(s)
}

\author{
Réflexions sur les usages de la prosopographie et de l'analyse \\ géométrique des données à partir de trois expériences de \\ recherche collective sur des objets transnationaux
}

\section{Frédéric LEBARON}

Frédéric Lebaron est professeur de sociologie à l'Université Versailles SaintQuentin-en-Yvelines et à l'IEP de Saint-Germain-en-Laye, membre du laboratoire Printemps (UMR 8085).

\begin{abstract}
A partir de plusieurs expériences collectives de recherche ayant pour point

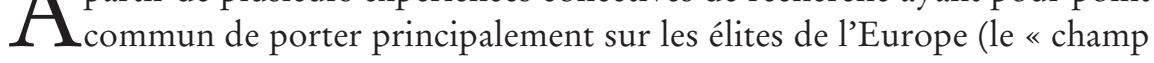
de l'Eurocratie » au sens le plus large ${ }^{1}$ ), on se propose dans le présent article d'adopter une posture réflexive à propos des enjeux et des problèmes de plusieurs étapes de ces enquêtes et de leur analyse : la conception du dispositif; la démarche de recueil d'information; la gestion de la base de données biographiques; les exploitations statistiques des données, en mettant en avant le recours aux outils et potentialités de l'analyse géométrique des données $(\mathrm{AGD})^{2}$.
\end{abstract}

On adopte donc ici un point de vue «méthodologique » et épistémologique, mais aussi proprement sociologique sur le processus qui conduit à exploiter des bases de données prosopographiques dans le cadre d'études transnationales, qui s'inscrivent dans une déjà riche tradition d'études de l'internationalisation des élites ${ }^{3}$. Cette réflexion, pour une part seulement per-

1. Georgakakis D. (dir.), Le champ de l'Eurocratie. Une sociologie politique du personnel de l'UE, Paris, Economica, 2012.

2. Cet article est le développement d'une présentation initialement esquissée à King's College à Londres, dans le cadre d'un séminaire organisé par Didier Bigo en 2014, et développée au Moulin d'Andé dans le cadre de l'école d'été thématique organisée par D. Georgakakis, J. Rowell et A. Vauchez en juin 2015.

3. Voir par exemple : Broady D., Palme M., Saint-Martin (de) M. (dir.), Les élites, formation, reconversion, internationalisation, colloque de Stockholm, 24-26 septembre 1993, Paris, Centre de sociologie de l'education et de la culture, École des hautes etudes en sciences sociales, 1995. Ce colloque, qui marque l'aboutissement de recherches menées au Centre de sociologie de l'éducation et de la culture, est aussi le point de départ de travaux prosopogra- 
sonnelle ${ }^{4}$, s'est nourrie d'expériences collectives d'assez longue durée, mises en ouvre par des équipes différentes, avec des perspectives distinctes, même si elles partagent des références théoriques, des problématiques et des façons de concevoir la démarche sociologique.

Elles ont en particulier en commun le recours à une perspective d'analyse relationnelle que résume la notion de «champ » issue des travaux de Pierre Bourdieu ${ }^{5}$, autour de laquelle de nombreuses discussions continuent de porter (voir par exemple le dossier de la revue Actes de la recherche en sciences sociales en décembre 2013 consacré au « champ 6 »).

Dans les trois cas évoqués, l'objectif de représenter statistiquement et visuellement le champ s'est imposé comme une étape centrale et importante pour formaliser et opérationnaliser cette notion, mais aussi pour poser les problèmes propres à chaque enquête de manière spécifique.

L'enjeu de l'analyse d'un champ transnational soulève de nombreuses difficultés : dans un contexte purement national, le système de formation et de sélection, les instances de consécration propres à un champ particulier sont relativement unifiés et faciles à identifier. Dès lors, les positions (sociales, institutionnelles...) sont plus faciles à mettre en relation les unes avec les autres, les « métriques »(en dehors de l'unité monétaire) étant relativement plus homogènes. Dans le contexte transnational, ici plus particulièrement européen, on est amené à faire l'hypothèse d'une relative unité des espaces étudiés sur des bases moins assurées, même si l'on verra que le processus d'homogénéisation d'élites transnationales peut dans certains cas être très avancé (comme on le voit avec la Banque centrale européenne).

La perspective adoptée, adossée à une démarche de sociologie quantitative fondée sur l'analyse géométrique des données, est aussi résolument associée à des démarches plus «qualitatives 7 ». L'analyse de trajectoires individuelles, qu'elle soit fondée ou non sur des entretiens et des observations directes, mobilisant divers documents d'archives, est en effet indissociable de l'ap-

phiques menés dans les années 1990, dans différents contextes nationaux : Brésil, Russie, Suède, etc.

4. Nous voudrions ici remercier tous les collègues qui ont été, à un moment où à un autre, à l'origine de telle ou telle réflexion et ont contribué à un titre ou à un autre à l'avancée de ces analyses.

5. En particulier, pour l'univers des élites : Bourdieu P., La noblesse d'État : grandes écoles et esprit de corps, Paris, Minuit, 1989.

6. Bourdieu J. (dir.), "Théorie du champ », Actes de la recherche en sciences sociales, $\mathrm{n}^{\circ} 200$ décembre 2013.

7. On s'appuie en particulier, en matière de démarche "qualitative », sur les réflexions développées par Olivier de Sardan : Olivier de Sardan J.-P., La rigueur du qualitatif. Les contraintes empiriques de l'interprétation socioanthropologique, Louvain-La-Neuve, Bruylant, 2008. Voir également, pour une réflexion historique et épistémologique profonde sur la démarche ethnographique : Weber F., Brève histoire de l'anthropologie, Paris, Flammarion, 2015. 
proche relationnelle des champs transnationaux. Le croisement des démarches (qualitatives et quantitatives) prend ici d'autant plus de sens qu'il s'agit non de raisonner dès le départ sur des « collectifs » (institutions, groupes) mais plutôt de restituer la complexité des parcours biographiques (ici principalement scolaires et professionnels).

\section{Les enquêtes}

\section{L'enquête sur les banques centrales (BANQCENT, 1999) 8}

Il s'agit d'une étude prosopographique portant sur les dirigeants de banques centrales (gouverneurs, sous-gouverneurs, membres des conseils), avec une focale particulière sur la Banque centrale européenne, plus précisément le Conseil des gouverneurs. Son dispositif, évolutif, a été élaboré dans le cadre de projets de recherche personnels postérieurs à notre thèse de doctorat et s'est développé depuis lors grâce à des collaborations techniques, notamment avec des étudiants en Maîtrise de sciences et techniques/Informatique et statistique appliquée aux sciences de l'homme (MST/ISASH) à l'université Paris Descartes, avec Karin Darin, post-doctorante au Centre universitaire de recherches sur l'action publique et le politique-Épistémologie des sciences sociales (CURAPP-ESS) et plus récemment Aykiz Dogan, doctorante en sociologie à l'Institut d'étude du développement économique et social (IEDES) à l'université Paris Panthéon-Sorbonne. Il s'agissait de comparer les parcours des banquiers centraux dans le monde, d'étudier la spécificité des banquiers centraux européens, d'analyser la relation entre leurs positions et leurs trajectoires d'une part, leurs prises de position d'autre part (orthodoxie monétaire, budgétaire, etc.). L'unité est ici quasi-« naturelle», puisque le Conseil des gouverneurs est l'instance centrale qui détermine la politique monétaire de la zone euro.

\section{L'enquête POLILEXES (2009)}

Émanant de sociologues du droit et des professions juridiques, ce projet visait à étudier l'espace des « professions » juridiques en Europe depuis le traité de Rome de 1957, dans la perspective de comprendre la contribution du droit à la construction européenne. L'enquête a été réalisée à partir de 2009 grâce à un financement de l'Agence nationale de la recherche (Projet Droit et juristes dans le gouvernement européen à I'Université Paris 1 Panthéon-Sorbonne, sous la direction de Guillaume Sacriste). L'équipe était principalement composée d'Antonin Cohen, Guillaume Sacriste, Antoine Vauchez et, pour les aspects plus méthodologiques, de Frédéric Lebaron, Antoine Mégie et Jérôme Pacouret. Elle a consisté en une série régulière de réunions de discussions qui ont permis d'avancer pas à pas durant plusieurs années et a donné lieu à divers colloques et publications. Dans ce travail,

8. Lebaron F., "Central bankers in the contemporary global field of power: a "social space" approach ", Sociological Review, vol. 56, Monograph 1, "Remembering Elites”, avril 2008, pp. 121-144 ; Lebaron F., « European Central Bank Leaders in the Global Space of Central Bankers: a Geometric Data Analysis Approach ", French Politics, vol. 8, n 3, 2010, pp. 294320 ; Lebaron F., «A Universal Paradigm of Central Banker? An inquiry based on biographical data ", Social Glance, vol. 1, n 1, octobre-décembre 2012, pp. 40-58; Lebaron F., «A Sociological Take on Central Bank Innovation », ECPR, General Conference, Université de Montréal, 28 août 2015. 
notre rôle a été celui d'un conseiller technique sur le plan du recueil de I'information et sur la perspective d'exploitations statistiques à l'aide des outils de l'analyse géométrique des données. La rédaction d'un ouvrage de synthèse est en cours.

Les enquêtes sur le champ de l'eurocratie (EUROCRATIE, 2014)

Le point de départ de ce travail est constitué par les recherches menées au sein du Groupe de sociologie politique européenne/Politique, religion, institutions et sociétés : mutations européennes (GSPE/PRISME) dans les années 2000, autour de Didier Georgakakis, Marine de Lassalle, et de plusieurs autres membres du GSPE/PRISME (V. Dubois, P. Juhem, V. Lozac'h, M. Mangenot, S. Michon, etc. 9). Elles ont conduit à la constitution d'une base de données prosopographiques sur le personnel de I'Europe. Ces enquêtes ont permis de documenter I'affirmation d'un capital européen et la structuration du champ de l'eurocratie. Willy Beauvallet et Sébastien Michon ont appliqué les méthodes d'analyse géométrique des données aux parlementaires européens 10.

En 2014, une enquête plus réduite a été lancée par Didier Georgakakis et nous-même, portant sur les transformations du recrutement social et professionnel de la haute administration bruxelloise (directeurs généraux, directeurs adjoints, etc.), notamment dans la période la plus récente. II s'agit d'étudier la montée en puissance du sous-champ de la gouvernance économique au sein du champ du pouvoir européen afin de comprendre, notamment, le maintien des politiques d'austérité budgétaire dans un contexte de chômage de masse et de faible dynamisme économique. La saisie des données a été réalisée par Florent Parruite (doctorant en science politique à l'université Paris-Ouest-Nanterre-La-Défense) en 2014 et $2015{ }^{11 .}$

\section{La conception du dispositif d'enquête}

À l'origine de toute enquête, des questions de recherche initiales conduisent à problématiser de la manière la plus claire possible les objectifs mêmes de l'enquête. Dans les trois cas analysés, de fortes problématisations sociologiques ou historiques avaient été développées préalablement à l'enquête " prosopographique », en relation avec un domaine d'études européennes et de sociologie politique internationale fortement constitué.

9. Georgakakis D., de Lassalle M., "Genèse et structure d'un capital institutionnel européen. Les très hauts fonctionnaires de la Commission européenne ", Actes de la recherche en sciences sociales, $\mathrm{n}^{\circ} 166-167,2007$, pp. 38-53.

10. Par exemple Beauvallet W., Michon S., «Faire carrière au Parlement européen : activation de dispositions et socialisation institutionnelle » in Georgakakis D. (dir.), Le champ de l'Eurocratie, Paris, Economica, 2012, pp. 13-42.

11. Georgakakis D., Lebaron F., "Le champ de la gouvernance économique européenne et les politiques d'austérité (2010-2015) : premières esquisses ", communication au colloque de Strasbourg " The economic crisis and the reconfiguration of european actors ", 4 novembre 2015. 
Cela a conduit à orienter fortement le questionnement dans des directions particulières, aussi bien du point de vue des individus enquêtés que des informations jugées pertinentes pour répondre à des questions spécifiques.

L'enquête sur les banques centrales partait, nous l'avons déjà souligné, de l'idée d'étudier la relation entre trajectoires professionnelles, caractéristiques sociales d'une part et «prises de position » d'autre part. Dans ce travail, l'Europe ne constituait en fait qu'un niveau de l'échelle d'observation parmi d'autres possibles. L'enquête sur le (sous-)champ des gouverneurs de la BCE visait avant tout, sur ce plan, à dégager les spécificités de l'espace européen dans une perspective "globale ", pour étudier seulement ensuite ses différenciations internes et leurs conséquences sur les orientations de la politique monétaire ${ }^{12}$. Le point de vue adopté sur la BCE consistait à l'envisager comme un sous-espace au sein d'un espace transnational plus global.

Dans l'enquête POLILEXES, le point de départ était un ensemble de questionnements autour du rôle structurant du droit dans la construction européenne, portant sur les caractéristiques et évolutions historiques des trajectoires biographiques dans un espace européen en cours de formation. L'enquête présentait dès le départ une forte dimension à la fois socio-historique et biographique, avec un fort intérêt pour les bifurcations, les marques d'hétéronomies, les trajectoires plurielles et hybrides, la structuration complexe du champ, qui passe d'une phase de juxtaposition et articulation d'histoires nationales autonomes à un espace plus clairement européanisé, notamment du fait même de la montée en puissance du droit communautaire.

L'enquête en cours sur le champ de l'eurocratie vise à poursuivre une analyse comparée des caractéristiques de certains groupes d'acteurs administratifs de la Commission européenne (directeurs, directeurs-adjoints, etc.) selon divers critères, pour établir empiriquement le renforcement tendanciel du sous-champ de la gouvernance économique européenne dans un espace plus large, là encore avec en arrière-plan l'existence d'un processus d'européanisation auquel contribuent tout particulièrement les «permanents » de l'Europe ${ }^{13}$. Deux périodes sont privilégiées, le début des années 2000 et le début des années 2010, alors que dans l'enquête originelle du GSPE/PRISME, la perspective historique et l'analyse systématique des trajectoires étaient beaucoup plus fortement affirmées, les données de carrière ayant été codées année par année. Il s'agit en effet de tester l'idée d'un renforcement avec la crise et d'une stabilité relative de la structure des carrières européennes, du côté des institutions économiques.

12. Lebaron F., "Banquiers ", in Lambert-Abdelgawad E., Michel H. (dir.), Dictionnaire des acteurs de l'Europe, Strasbourg, Larcier, 2014.

13. Voir Georgakakis D. (dir.), Le champ de l'Eurocratie. Une sociologie politique du personnel de l'UE, op. cit. 


\section{Le plan de recueil de l'information et la délimitation de l'espace étudié}

Les premiers choix de recueil d'informations ont une dimension théorique, souvent peu consciente des spécialistes d'un domaine particulier. La définition des limites de l'espace ou du champ étudié est, sans aucun doute, l'enjeu le plus débattu et le plus critique, dans la mesure où elle conduit à abandonner le flou relatif des concepts pour en proposer une première opérationnalisation, qui est aussi une mise à l'épreuve empirique radicale et peut susciter des formes de rejet immédiat. On peut illustrer les problèmes posés par cette étape dans chaque cas, avec comme point commun le fait que ces choix, dans la mesure où ils « calibrent » fortement les potentialités empiriques des données, sont de nature théorique autant que pratique.

Dans les trois études présentes, l'enjeu consiste au moins pour une part à affirmer l'existence d'un espace européen suffisamment autonome par rapport aux espaces nationaux et suffisamment spécifique par rapport à l'espace global : cette double distinction ne va jamais de soi, certains champs pouvant être caractérisés par une forte autonomie nationale, d'autres, à l'opposé, par une véritable insertion «globale ». Plutôt que de disserter sans fin sur les jeux d'échelle ou l'articulation multi-niveau, il s'agit de caractériser le degré d'autonomie de chaque niveau pertinent d'analyse.

Dans l'étude sur les banquiers centraux, c'est la notion même de direction (ou gouvernance) d'une banque centrale qui est en jeu. En étendant la définition du sous-champ pertinent des gouverneurs aux sous-gouverneurs et membres des conseils de politique monétaire, on a voulu mettre l'accent sur le critère institutionnel qui fait des conseils de politique monétaire le véritable "souverain » en la matière. Les acteurs retenus sont des acteurs décisionnels directs, qui «votent » ou, au moins, prennent position officiellement sur les décisions monétaires. Le caractère très centralisé, très formalisé et très uniformisé du secteur rend possible cette séparation, en particulier par rapport aux dirigeants de l'administration de la Banque centrale, qui sont donc considérés ici, à tort ou à raison, comme des « exécutants ». La spécificité de la BCE conduit à s'intéresser particulièrement au sous-espace constitué par l'exécutif européen en matière monétaire, à savoir le Conseil des gouverneurs, composé de membres du directoire et de gouverneurs de banques centrales nationales. L'enquête couvre une période qui va de 1999 à aujourd'hui et se situe à l'échelle mondiale. La base compte actuellement $\mathrm{N}=660$ individus. Des zones sont beaucoup moins bien couvertes que d'autres et la liste des membres des conseils n'est pas toujours facile à obtenir, sans parler bien sûr des CV des membres, qui sont très inégalement et variablement renseignés.

L'enquête POLILEXES s'est restreinte aux professionnels du droit, dans une acceptation cependant relativement large : juges aux différentes cours, 
référendaires, parlementaires spécialisés dans le droit (ce qui s'objective par leur appartenance à des commissions juridiques du parlement), fonctionnaires juristes de la CE depuis le traité de Rome. Trois grandes périodes (délimitées par chaque « grand élargissement » avant celui de 2004) ont été intégrées. À plusieurs reprises, le problème des avocats, non pris en compte en tant que tels mais présents lorsqu'ils ont occupé l'une des positions retenues, a été soulevé. Faute de sources comparables et pour des raisons pratiques, ce groupe de taille considérable n'a pas été intégré à la base en son état actuel ( $\mathrm{N}=530)$. L'unité européenne est, on le voit, problématique dans une perspective d'histoire relativement longue, qui voit se succéder des « groupes de pays » de plus en plus larges, ce qui a même justifié le choix de ne pas prolonger la même analyse après 2004, soit l'élargissement qui a le plus clairement mis en cause l'homogénéité de l'ensemble constitué par l'UE.

Dans le cas de l'enquête EUROCRATIE, on s'est concentré sur les directeurs, membres de cabinets, de la haute administration des institutions européennes (avec une focale sur les directions dites «économiques ", ce qui a fait l'objet de discussions, notamment s'agissant de la direction du budget), en laissant donc de côté (ce qui ne va pas de soi) les directions «économiques » nationales des ministères des finances ou les banques centrales nationales aux niveaux inférieurs au gouverneur. On s'est donc concentré sur la comparaison de deux dates (2001 et 2012-13) et sur le cœur «administratif » de l'eurocratie $(\mathrm{N}=410)$, et sur la partie des élites européennes qui est la plus strictement « européenne » dans la mesure où elle n'appartient pas à une administration nationale. Ce choix se justifie dans une large mesure par l'autonomisation accrue du champ de l'eurocratie, dont l'inertie propre contribue pour une part à celle des dynamiques européennes.

On a donc des informations couvrant des domaines d'ampleur et de nature très différentes : nombre de variables, avec environ quarante pour BANQCENT, ce qui est très lié aux sources, plus de cinq cents pour POLILEXES (ce qui s'explique par des duplications avec trois périodes distinctes, la volonté d'exhaustivité des informations professionnelles recueillies...), et seulement trente variables pour EUROCRATIE (l'idée étant de disposer d'un petit modèle réduit et de ne pas exagérer le degré de précision de l'information biographique nécessaire pour analyser les structures).

L'organisation et la division du travail scientifique ont aussi reposé sur des modèles différents d'organisation, du plus artisanal et individuel (BANQCENT) au plus standardisé et collectif (POLILEXES), du plus "indivis » (BANQCENT) au plus segmenté (POLILEXES). Ces nuances entrainent des différences de temporalités, de cumulativité possible, de nature des «outputs». 


\section{Fabrication et gestion de la base de données}

On a eu recours dans ces trois enquêtes à des sources diverses : annuaires, sites web, etc. L'enquête EUROCRATIE s'est appuyée jusqu'ici essentiellement sur le Trombinoscope. La nécessité de croiser les sources est fondamentale et le travail de construction de l'information est ici quasi-infini, au risque de susciter l'épuisement et la frustration des chercheurs, perceptibles dans certaines de ces enquêtes.

Les sources biographiques transnationales sont complexes à manipuler : soit on s'en tient à des annuaires internationaux (comme les annuaires officiels, les Who's Who internationaux, le Trombinoscope, etc.), ce qui implique des informations relativement standardisées et faciles à coder, mais relativement superficielles, soit on mobilise un maximum d'informations nationales, avec des degrés de précision très variables selon les régions, les institutions, etc. En particulier, l'accès aux informations sur la famille et l'origine sociale est souvent difficile à obtenir alors même que l'on peut penser que les caractéristiques issues de l'habitus primaire des individus en matière culturelle (multilinguisme, familiarité avec les institutions transnationales...) sont très structurantes de leurs expériences.

Divers problèmes juridiques, que nous n'évoquerons pas ici, sont liés à la constitution de fichiers de données qui, par définition, ne sont pas anonymisés mais reposent sur des informations uniquement publiques.

Dans les trois enquêtes, on a eu recours à des procédures manuelles menées collectivement, échelonnées dans le temps, parfois déléguées à des personnes non impliquées dans l'élaboration initiale (cas de BANQCENT et EUROCRATIE). De plus en plus, l'automatisation de la construction de l'information tire parti de la généralisation et de la facilité d'accès des outils numériques (comme le montrent les travaux menés par François Denord, Paul Lagneau-Ymonet et Sylvain Thine ${ }^{14}$ ).

Des sous-populations spécifiques ajoutent une difficulté supplémentaire. On doit ainsi faire face à des situations contrastées du point de vue de l'accès à l'information, selon le groupe, la région du monde. Beaucoup de «nonréponses » (réponses manquantes) sont le lot du chercheur en prosopographie contemporaine et c'est particulièrement vrai dans le contexte transnational, d'où l'intérêt de méthodes comme l'analyse des correspondances multiples spécifiques qui permet de traiter le problème des non-réponses de façon simple et élégante.

14. Denord F., Lagneau-Ymonet P., Thine S., «Le champ du pouvoir en France. », Actes de la recherche en sciences sociales, $\mathrm{n}^{\circ} 190,2011$, pp. 24-57. 
Les premiers tris à plat font apparaitre des variables comprenant beaucoup de modalités à faibles ou très faibles effectifs, ce qui renvoie à la richesse quasiethnographique de l'information collectée par des spécialistes attentifs aux fines différenciations au sein d'un champ. Mais ce grand nombre de modalités à faible fréquence des variables recueillies implique rapidement l'enjeu du codage et de ses problèmes. On va alors devoir passer du qualitatif au modélisé pour revenir au qualitatif dans un second temps, au moment de l'interprétation des structures des espaces mis au jour.

La question des « prises de position » et des « discours » reste, enfin, un enjeu important dans le travail de construction de données biographiques. Comment les intégrer dans le recueil d'information ? De notre point de vue, il s'agit avant tout de coder l'information comme on code des informations plus «objectives », en étant toujours attentif aux caractéristiques des prises de position : leur caractère contextuel, leur variabilité, la difficulté d'interpréter les discours, etc. On peut s'inspirer partiellement ici des démarches utilisées, par exemple, en analyse de réseaux pour analyser les co-citations d'articles ${ }^{15}$.

Le succès du «Big Data » va sans aucun doute permettre à l'avenir de traiter des bases de données sur des échelles beaucoup plus vastes, avec une visée plus exhaustive, mais sans résoudre aucun des problèmes méthodologiques et scientifiques fondamentaux évoqués dans cet article : beaucoup de " cases vides ", d'hétérogénéité dans l'information collectée vont même sans doute accentuer les problèmes de pertinence sociologique déjà occasionnés par la lourdeur et les méandres du travail prosopographique.

On a ainsi un processus continu de recueil, codage, premiers traitements, qui semble à certains égards initier une quête sans fin. L'exemple de POLILEXES nous a ainsi fait prendre régulièrement la mesure des limites de toute base de données ambitieuse : à la faveur des « surprises », des « erreurs » détectées, renvoyant parfois à l'application par chaque codeur de normes non explicitées auparavant et pas toujours totalement harmonisées, a pu se faire jour une nouvelle volonté d'harmonisation, mais aussi une forme de relativisation des données numériques produites.

Le codage apparaît rapidement comme une nécessité pour modéliser mais aussi comme perte d'information potentielle, éventuellement provisoire, pour la mise en forme et la représentation des résultats. La qualité des analyses statistiques, en particulier géométriques, dépend fortement de celle des codages effectués, qui dépendent pour une large part de l'expertise des experts, seuls aptes, par exemple, à déterminer les bonnes divisions concernant l'espace des établissements universitaires.

15. Callon M., Courtial J.P., Pénan H., La scientométrie, Paris, PUF, 1993. 
On peut, en pratique, comparer les sous-populations à partir d'indicateurs (sex-ratio, proportion des différentes classes d'âge, etc.), ce qui est à la fois une démarche heuristique, mais aussi un outil de vérification de la qualité des données recueillies. L'intérêt de la comparaison systématique de populations transnationales est, précisément, de les dés-essentialiser en les caractérisant par des traits banals et immédiatement comparables, comme un sex-ratio, une proportion de titulaires du doctorat, la part des disciplines de formation initiales, la présence de «passages obligés » dans les carrières, etc. Le raisonnement en termes de sous-populations, inspiré de la socio-démographie ${ }^{16}$, complète ainsi l'approche fondamentalement biographique associée à l'AGD.

\section{L'analyse géométrique des données comme cadre pour l'exploration d'es- paces transnationaux}

La base de données «nettoyée " n'est que le début d'un processus de recherche systématique, qui n'est pas forcément toujours mené par les concepteurs d'origine. Une étape décisive est toujours la statistique descriptive élémentaire. Dans les trois cas, un premier ensemble de résultats se présentent sous la forme d'informations de synthèse sur une population (voir, par exemple, les tableaux sur les banquiers centraux européens dans les articles cités). Le recours à des tableaux de contingence permet d'affiner les comparaisons de sous-populations et d'obtenir des résultats faciles à communiquer et à interpréter.

Les analyses «multivariées » les plus diverses peuvent alors être menées sur ce type de base de données. Elles vont jusqu'à l'utilisation des méthodes permettant de construire des classes de trajectoires : la classification ascendante hiérarchique (CAH) prolongeant l'analyse des correspondances multiples (ACM) ou l'analyse de séquence, etc. Les données de co-appartenance permettent de construire des graphes de réseaux sociaux et d'interpréter les centralités, intermédiarités, etc.

Nous avons privilégié ici le recours à l'analyse géométrique des données comme instrument de modélisation géométrique des espaces sociaux ${ }^{17}$. Il s'agissait avant tout de répondre aux questions de recherche des concepteurs de l'enquête ou à des questions que les données permettaient de commencer à résoudre. La construction d'un espace n'est qu'une étape de l'analyse sociologique, qui permet surtout de remplacer des questionnements flous par une opérationnalisation systématique, laquelle oblige à discuter de points de choix fondamentaux et impose de calibrer ses analyses, tant s'agissant de l'importance des effets et des variations que de « principes de division » qui traversent

16. Fouquet A., Vinokur A., Démographie socio-économique, Paris, Dalloz, 1996.

17. Lebaron F., L'enquête quantitative en sciences sociales : recueil et analyse des données, Paris, Dunod, 2006 ; Lebaron F., Le Roux B. (ed.), La méthodologie de Pierre Bourdien en action. Espace culturel, espace social et analyse des données, Paris, Dunod, 2015. 
tout univers social. Dans le contexte de l'étude d'espaces transnationaux, cette démarche permet de substituer à des entités abstraites qui risquent toujours de devenir des essences sui generis des espaces multidimensionnels reposant sur la relation statistique s'établissant entre les propriétés pertinentes des individus.

Ce recours heuristique et pragmatique n'implique pas de renoncer à la rigueur de la formalisation et de la pratique statistique mais de les concevoir comme des instruments de construction progressive de l'objet scientifique, susceptibles de nourrir le dialogue avec les observations plus « qualitatives » et d'affiner les stratégies interprétatives ou «modélisatrices».

Pour illustrer cet usage, on présente successivement trois exemples de construction d'espace, en insistant sur leur caractère heuristique, les problèmes posés et les limites ${ }^{18}$.

\section{Exemple 1. Le cas des innovations au sein de la BCE}

On commence par présenter l'étude de cas des « innovations » au sein de la BCE. L'étude exploratoire vise surtout à montrer les potentialités heuristiques de cette démarche, s'agissant de l'étude de prises de position dans un sous-champ particulier.

La population est constituée ici des 38 membres du conseil des gouverneurs de la BCE présents entre janvier 2010 et décembre 2014. En dehors des variables socio-démographiques, on a codé le style « économique et monétaire » des membres du conseil sur la base des prises de position explicites et publiques et des commentaires et codages effectués par les European Central Bank watchers sur leurs sites internet.

L'ACM spécifique a été effectuée sur 12 questions actives et 31 modalités actives, afin de modéliser géométriquement l'espace social du conseil durant la période : âge, sexe, position à la BCE entre 2010 et 2014, niveau de diplôme, discipline, dominante de la carrière, passages par la politique, la Banque centrale, l'université, la fonction publique, la finance.

L'étude des valeurs propres révèle un fort premier axe, suivi de deux axes de moindre «importance » en tant que résumés des données (soit un taux modifié de $75 \%$ environ). On se contentera ici d'étudier les deux premiers axes.

18. Les analyses ont toutes été réalisées avec le logiciel SPAD8, et le calcul des taux modifiés avec la Macro Excel réalisée par Flora Chanviil. 


\begin{tabular}{|c|c|c|c|c|c|}
\hline Numéro & Valeur propre & $\begin{array}{c}\text { Taux brut } \\
\text { de variance }\end{array}$ & $\begin{array}{c}\text { Taux brut } \\
\text { de variance } \\
\text { cumulé }\end{array}$ & $\begin{array}{c}\text { Taux } \\
\text { de variance } \\
\text { modifié }\end{array}$ & $\begin{array}{c}\text { Taux modifié } \\
\text { cumulé }\end{array}$ \\
\hline 1 & 0,254 & 16,0 & 16,0 & $45,1 \%$ & $45,1 \%$ \\
\hline 2 & 0,178 & 11,2 & 27,1 & $17,3 \%$ & $62,3 \%$ \\
\hline 3 & 0,160 & 10,1 & 37,2 & $12,8 \%$ & $75,1 \%$ \\
\hline 5 & 0,149 & 9,4 & 46,6 & $10,3 \%$ & $85,4 \%$ \\
\hline
\end{tabular}

Tableau 1 : Valeurs propres, taux de variance bruts, taux modifiés

Le graphique 1 montre le nuage des modalités (les $30 \%$ les plus contributives) dans le premier plan principal. À droite, on trouve parmi les modalités les plus contributives : «carrière principale dans l'administration » (« main career in an administration»), Master, a été membre du Directoire entre 2010 et 2014 («being a member of the executive board at a moment during the period 2010-2014»), femme, droit, administration et politique. À gauche, on trouve les modalités opposées (université, secteur privé, pas de passage par l'administration...).

Le premier axe oppose donc des carrières politiques et administratives à des carrières soit académiques, privées ou internes à la Banque centrale. C'est un axe de capital politique et bureaucratique, ce qui est lié à la jeunesse au sein du conseil des gouverneurs et donc à un processus générationnel et genré.

Le deuxième axe oppose les carrières externes, dans le secteur privé ou les universités, aux carrières internes à la Banque centrale, montrant un clair principe de distinction au sein de l'espace.

La projection d'éléments supplémentaires (graphique 2) montre que le premier axe est descriptivement lié à une opposition entre «bawks » (faucons, hostiles à l'assouplissement monétaire et à l'innovation) et " modérés » à droite, et «doves » (colombes, favorables à l'assouplissement), plus clairement à gauche (avec un écart de 0,59 écarts-types, lisibles directement à l'aide des coordonnées dans le nuage des modalités, entre les deux modalités extrêmes). L'absence de capital politique et bureaucratique, la relation avec le secteur privé et l'université semblent être globalement associés à une orientation plus « innovatrice », plus « modérée » ou «pragmatique ».

Cette division permet d'entrevoir les raisons des difficultés internes au Conseil des gouverneurs et l'existence d'un soutien fort à l'orientation « innovatrice » de Mario Draghi en matière monétaire. Celui-ci illustre par sa propre trajectoire hybride une structure mixte de capital, associée à une conception pragmatique de la politique monétaire. 


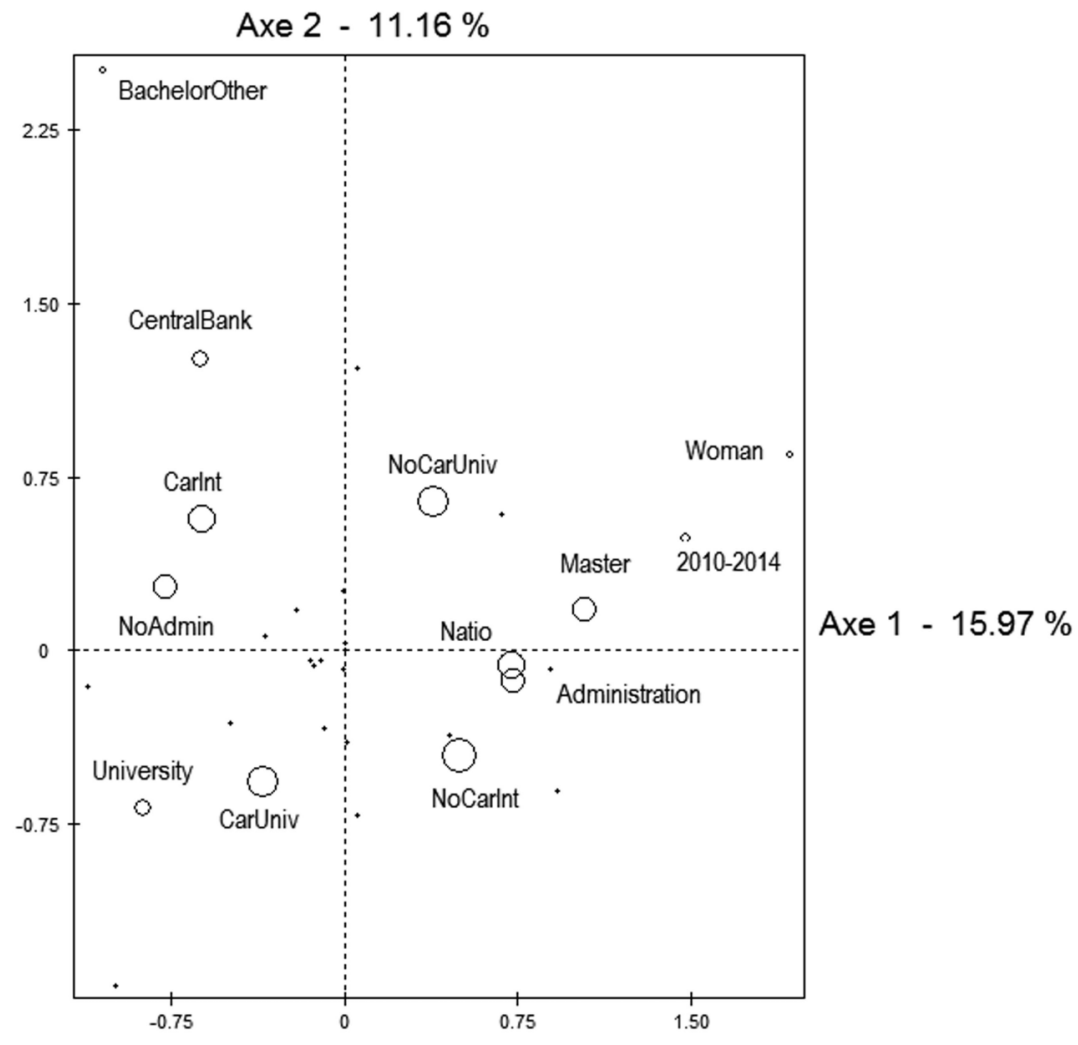

Graphique 1 : modalités actives les plus contributives dans le plan 1-2 (30\% de l'ensemble des modalités)

Le nuage des individus (graphique 3) montre aussi une forte dispersion au sein de chacun des deux sous-groupes de «faucons » et de «colombes » et de fortes divisions au sein du capital politique et bureaucratique. Les membres les plus « récents » du Directoire, qu'ils soient plutôt colombes (B. Coeuré) ou plutôt faucons (S. Lautenschläger), sont clairement situés à droite sur le premier axe. On peut s'attendre à ce que leur « influence » politique soit de forte magnitude.

L'étude montre finalement les divisions politiques internes du Conseil des gouverneurs, qui sont liées aux caractéristiques sociales et professionnelles de ses membres. Au pôle de la résistance à l'innovation, on trouve notamment les banquiers centraux allemands (mais pas tous, des personnes comme Jörg Assmussen étant plutôt des colombes), des acteurs comme le Luxembourgeois Yves Mersch ou le Finlandais Erkki Liikanen, membres d'une « coalition germanique » au sein du conseil. À l'opposé, le groupe soutenant Mario Draghi par ses déclarations et ses votes est mené par un petit groupe d'acteurs à fort capital bureaucratique et politique, mais aussi par une majorité (silencieuse sur 


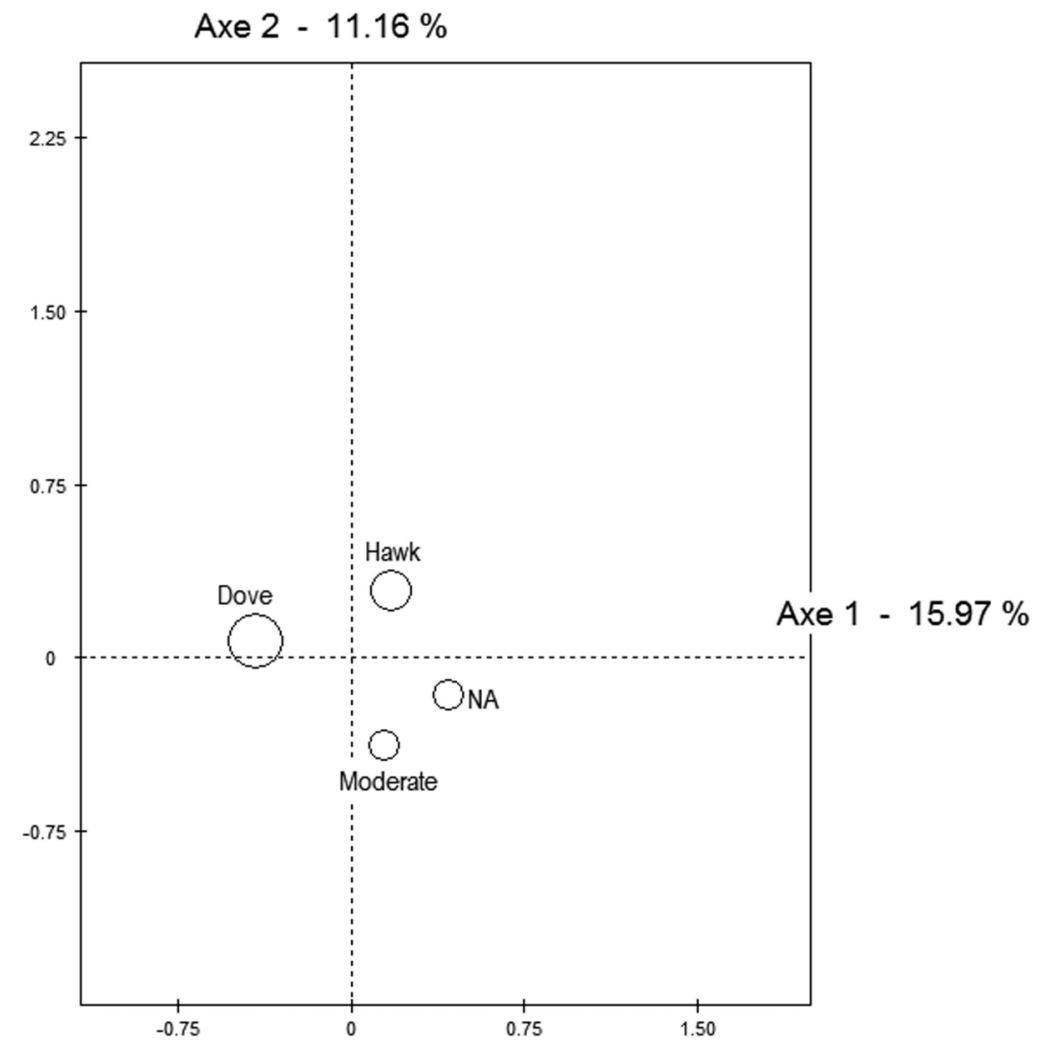

Graphique $2:$ : doves» (colombes) versus « hawks» (faucons) (éléments supplémentaires dans le nuage des modalités)

beaucoup de sujets) de gouverneurs nationaux, plus souvent issus des universités et du secteur privé. Construire et garder une majorité est clairement un enjeu fort au sein du Conseil en période de fortes turbulences monétaires et financières.

Notons que les résultats reposent ici sur de très faibles effectifs, ce qui implique de les prolonger par des procédures d'inférence, en particulier d'inférence combinatoire qui évite le recours à des « assomptions » trop rigides ${ }^{19}$.

L'analyse conduit ainsi à établir l'importance de propriétés de trajectoires définies à l'échelle de l'espace européen des banquiers centraux, contre la réduction des prises de position au poids de la seule variable nationale.

19. Le Roux B., Rouanet H., Geometric Data Analysis. From Correspondence Analysis to Structured Data Analysis, Dordrecht, Kluwer, 2004. 


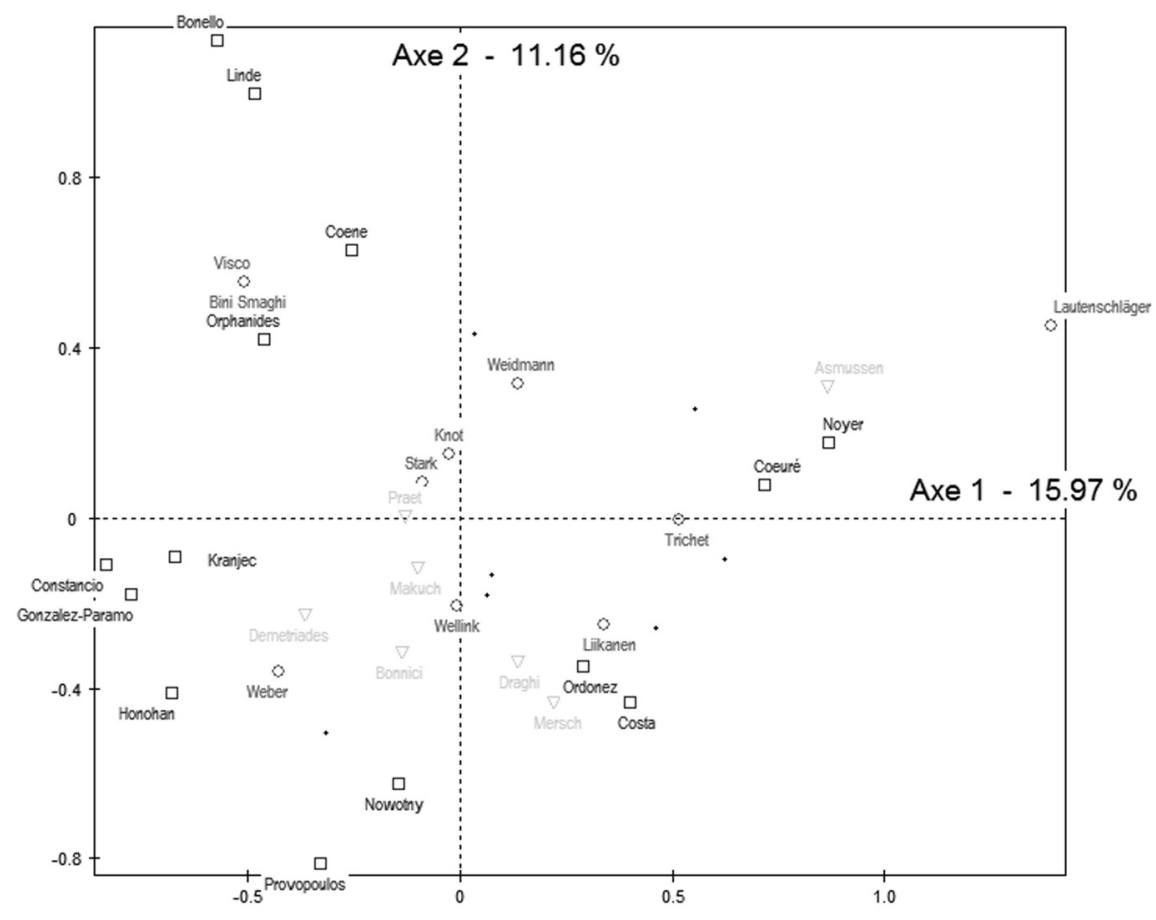

Graphique 3 : nuage des individus avec leurs prises de position (carrés : "doves », cercles : " hawks », triangles : " moderates »)

Exemple 2. L'espace des professions juridiques européennes des années 1950 à 2004

On a choisi dans ce cas de construire un espace des professions juridiques européennes des années 1950 à 2004 en s'appuyant sur les informations relatives aux individus concernant les questions analysées plus haut. Il s'agissait d'étudier dans quelle mesure la structure générale de l'espace européen ainsi construite est reliée à des variables telles que la période d'activité, les positions occupées, ou toute autre information contenue dans la base. Cette étape du travail permet de dégager quelques résultats importants, en faisant apparaître une dynamique globale d'internationalisation des formations, plus prononcée dans certains secteurs de l'espace que dans d'autres, et en faisant apparaître les principales polarisations internes à cet univers, déjà avancées dans divers travaux sur le sujet 20 .

20. Cohen A., «L'Europe en constitution. Professionnels du droit et des institutions entre champ académique international et "champ du pouvoir européen” ", in Cohen A., Lacroix B., Riutort P., Les formes de l'activité politique. Éléments d'analyse sociologique, du XVIII siècle à nos jours, Paris, Presses Universitaires de France, 2006, pp. 297-315 ; Cohen A., Vauchez A., "Sociologie politique de l'Europe du droit », Revne française de science politique, vol. 60, $\mathrm{n}^{\circ}$ 2, 2010, pp. 223-226 ; Mégie A., Sacriste G., « Polilexes : champ juridique européen et polity communautaire ", Politique européenne, $\mathrm{n}^{\circ} 28,2009$, p. 157-162 ; Vauchez A., Witte (de) B., The European Legal Field, Oxford, Hart Publishing, 2012. 
L'espace européen analysé est constitué de 442 individus et construit à partir de 15 questions actives et 45 modalités actives relatives à trois domaines :

- deux questions (quatre modalités actives) relatives à l'identité sociodémographique : sexe [deux modalités] et type de pays [deux modalités : grand et petit];

- six questions (treize modalités actives) relatives à la trajectoire scolaire et académique : niveau de diplôme [cinq modalités dont deux passives" : Doctorat, Master, Licence, Bac* et NSP*], lieu du diplôme [quatre modalités dont deux passives" : partiellement à l'étranger, entièrement national, entièrement à l'étranger*, NSP*], université aux États-Unis [deux modalités], collège de Bruges [deux modalités], université de Paris [deux modalités], Oxbridge [deux modalités];

- sept questions sur la trajectoire professionnelle (vingt-huit modalités actives) : âge de premier accès à une institution européenne [trente modalités dont une passive ${ }^{*}$ : avant 30 ans, 30-39, 40-49, 50-59, 60 et plus, NSP*], secteur de la première profession [six modalités : académique, administratif, économique, judiciaire, politique, autre (association, journalisme)], secteur de la dernière profession [sept modalités dont deux passives (autre et NSP*)], nombre de secteurs traversés sur l'ensemble de la carrière [quatre modalités : 1, 2, 3 , et 4-5 secteurs], nombre de positions dans les institutions européennes (cinq modalités, dont une passive*: 1, 2, 3-4, 5 et plus, NR*], passage par le secteur privé [deux modalités], passage par la politique [deux modalités].

L'espace est donc majoritairement construit à partir des indicateurs de trajectoire scolaire et surtout professionnelle, ce qui correspond bien à la perspective de la recherche, centrée sur la différenciation des parcours professionnels interne au champ juridique européen. L'analyse suppose un minimum d'homogénéité des variables actives retenues, pour que la comparaison soit possible, et fait en même temps l'hypothèse que les différenciations relatives à ces variables sont signifiantes à l'échelle de l'espace européen.

\begin{tabular}{|c|c|c|c|c|c|}
\hline Numéro & Valeur propre & $\begin{array}{l}\text { Taux brut } \\
\text { de variance }\end{array}$ & $\begin{array}{l}\text { Taux brut } \\
\text { de variance } \\
\text { cumulé }\end{array}$ & $\begin{array}{c}\text { Taux } \\
\text { de variance } \\
\text { modifié }\end{array}$ & $\begin{array}{l}\text { Taux modifie } \\
\text { cumulé }\end{array}$ \\
\hline 1 & 0,184 & 9,1 & 9,1 & $49,8 \%$ & $49,8 \%$ \\
\hline 2 & 0,124 & 6,1 & 15,2 & $14,1 \%$ & $63,9 \%$ \\
\hline 3 & 0,110 & 5,4 & 20,6 & $8,8 \%$ & $72,7 \%$ \\
\hline 4 & 0,107 & 5,3 & 25,9 & $7,9 \%$ & $80,5 \%$ \\
\hline 5 & 0,100 & 4,9 & 30,8 & $5,8 \%$ & $86,3 \%$ \\
\hline
\end{tabular}

Tableau 2 : Valeurs propres, taux de variance bruts et taux modifiés des cinq premiers axes 
On a mené une ACM spécifique, dont l'examen des résultats conduit à retenir quatre axes (dont nous nous contenterons d'étudier les deux premiers ici).

L'axe 1 (étudié à partir des $30 \%$ de modalités les plus contributives) est de façon prédominante un axe de trajectoire professionnelle et scolaire. Il oppose :

- du côté positif, pas de passage par le secteur politique (11,6\%), cursus national et à l'étranger $(9,7 \%)$, université aux États-Unis (7,8\%), collège de Bruges (5,8\%), 1 secteur sur l'ensemble de la carrière (5\%), entrée avant 30 ans $(3,7 \%)$;

- du côté négatif, dernière profession politique $(11,7 \%)$, passage par le secteur politique $(10,4 \%)$, cursus strictement national (4,1\%), entrée dans les institutions entre 50-59 ans (3,1\%).

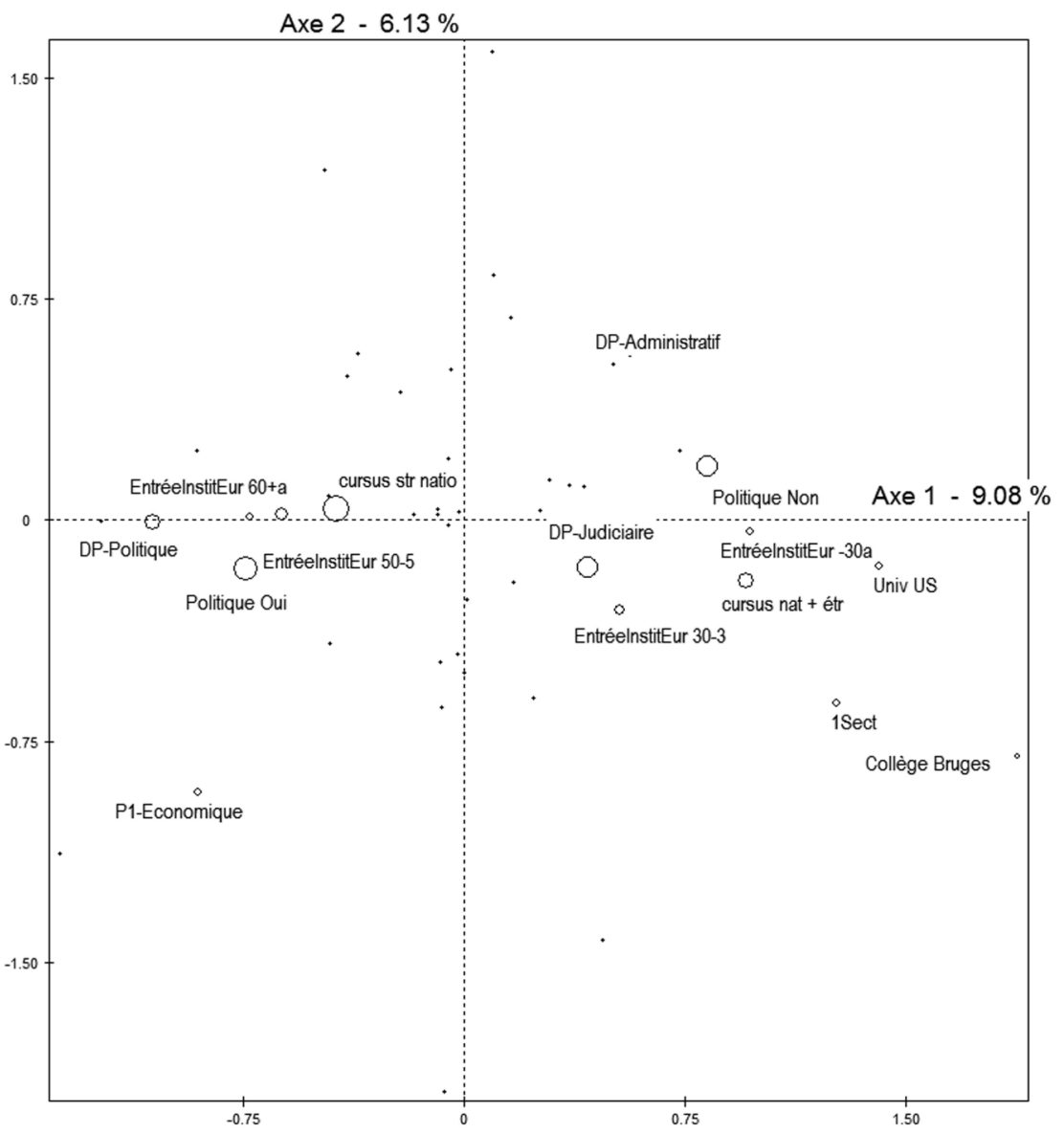

Graphique $4: 30 \%$ des modalités les plus contributives sur l'axe 1 
C'est donc un axe fortement déterminé à la fois par le lien à la politique et par les trajectoires académiques, qui peuvent être menées dans certains pays et institutions internationales ou au contraire "strictement nationales ». Il oppose donc le capital académique national à un certain type de capital académique international et le capital politique aux autres types de capitaux.

L'axe 2 est un axe distinguant des types de trajectoire professionnelle. Il oppose :

- du côté négatif (en bas du graphique) : secteur privé (12,2 \%), dernière profession économique $(7,8 \%)$, master $(7,8 \%)$, Oxbridge $(7,4 \%)$, première profession judiciaire $(5,1 \%)$, première profession économique $(3,8 \%)$;

- du côté positif (en haut du graphique) : pas de secteur privé (16\%), doctorat $(5,8 \%)$ première profession académique $(5,7 \%)$, deux positions européennes $(3,2 \%)$, première profession administrative $(2,6 \%)$, dernière profession administrative $(2,5 \%)$, première profession association-journalisme $(2,4 \%)$.

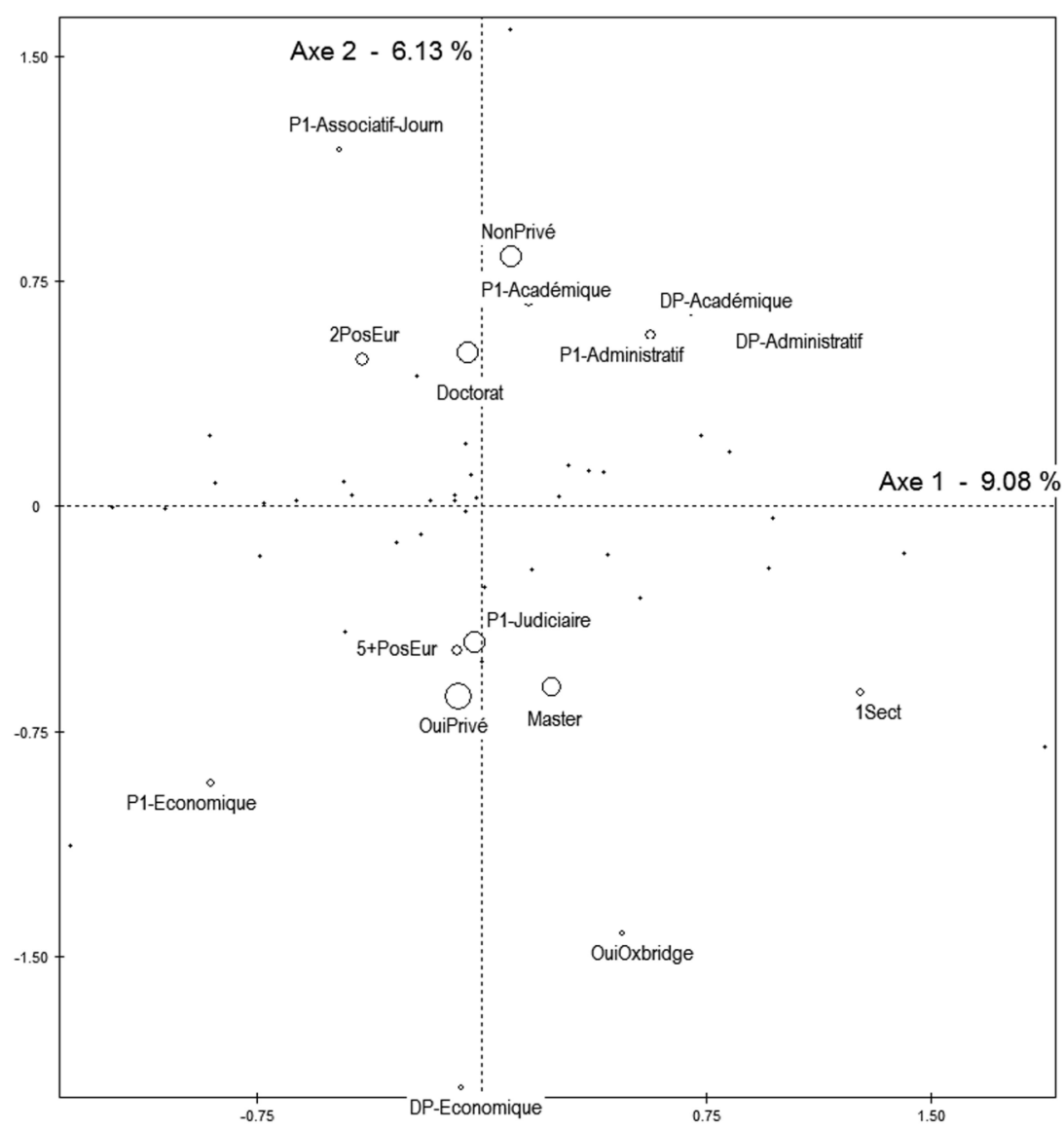

Graphique $5: 30 \%$ des modalités les plus contributives sur l'axe 2 
L'axe 2 oppose clairement un pôle privé et économique en bas à un pôle public et académico-administratif en haut.

Les axes 3 et 4 s'interprètent également assez aisément ${ }^{21}$. L'axe 3 est un axe de capital européen, opposant à gauche un fort capital européen, des trajectoires associatives et journalistiques ou encore administratives, le passage par le collège de Bruges, la précocité dans les institutions européennes et, à droite, des trajectoires dans le secteur judiciaire, entrée tardive dans le champ. L'axe 4 oppose les trajectoires multi-sectorielles, souvent liées aux mondes académique et économique, en haut, aux trajectoires mono-sectorielles, plus souvent liées au monde politique, en bas.

Le nuage des individus permet de visualiser les individus " parangons", individus typiques d'un pôle de l'espace.

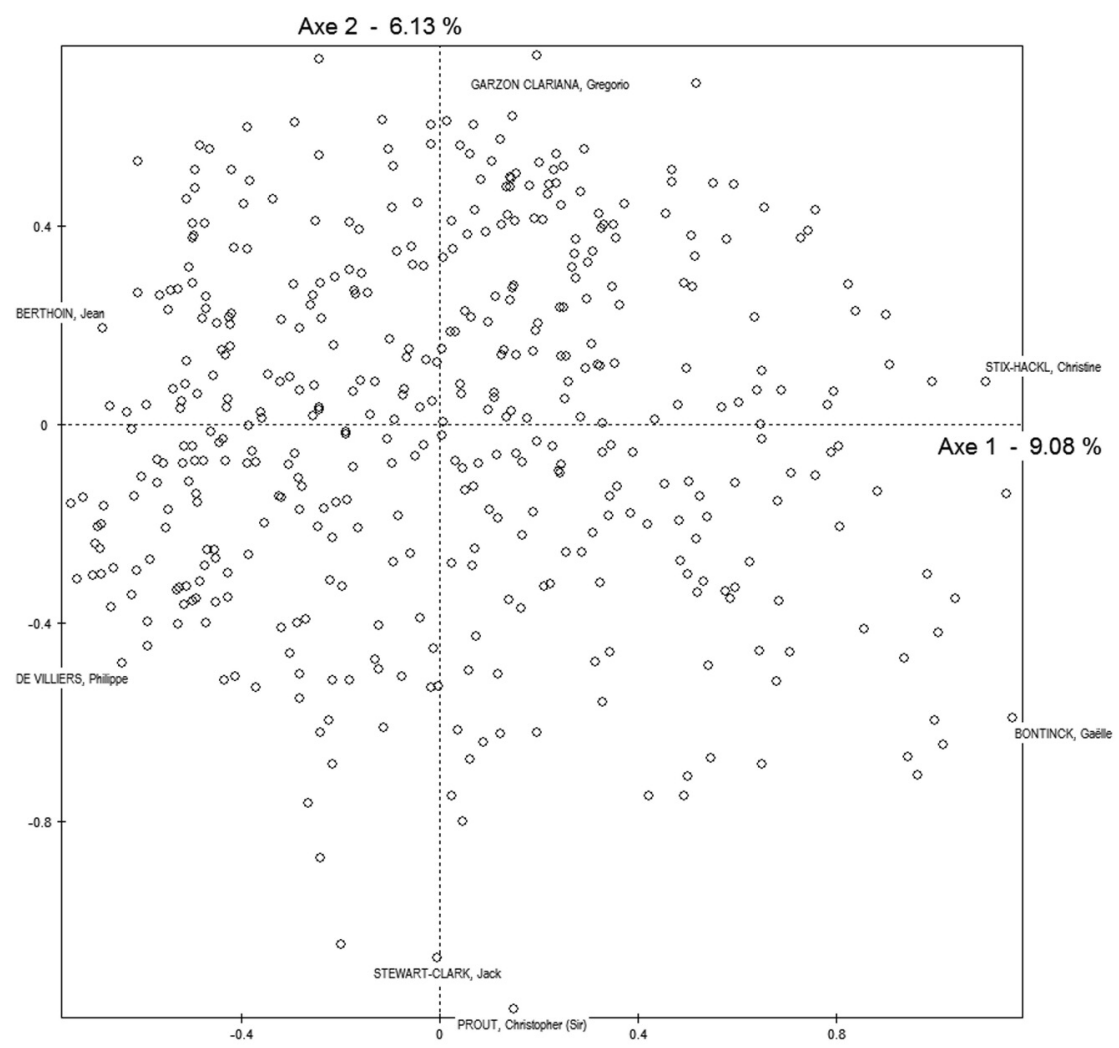

Graphique 6 : Nuage des individus dans le plan 1-2

21. Nous ne les représentons pas ici. 
On observe sur le premier axe une nette opposition entre les parlementaires à gauche, les référendaires, membres des services juridiques et membres du tribunal de la fonction publique à droite, les juges occupant une position intermédiaire.

Sur l'axe 2, une opposition entre juges (en haut) et membres du tribunal de la fonction publique (en bas) apparaît. Pour le reste, on trouve peu de différences entre les diverses «populations » sous ce rapport.

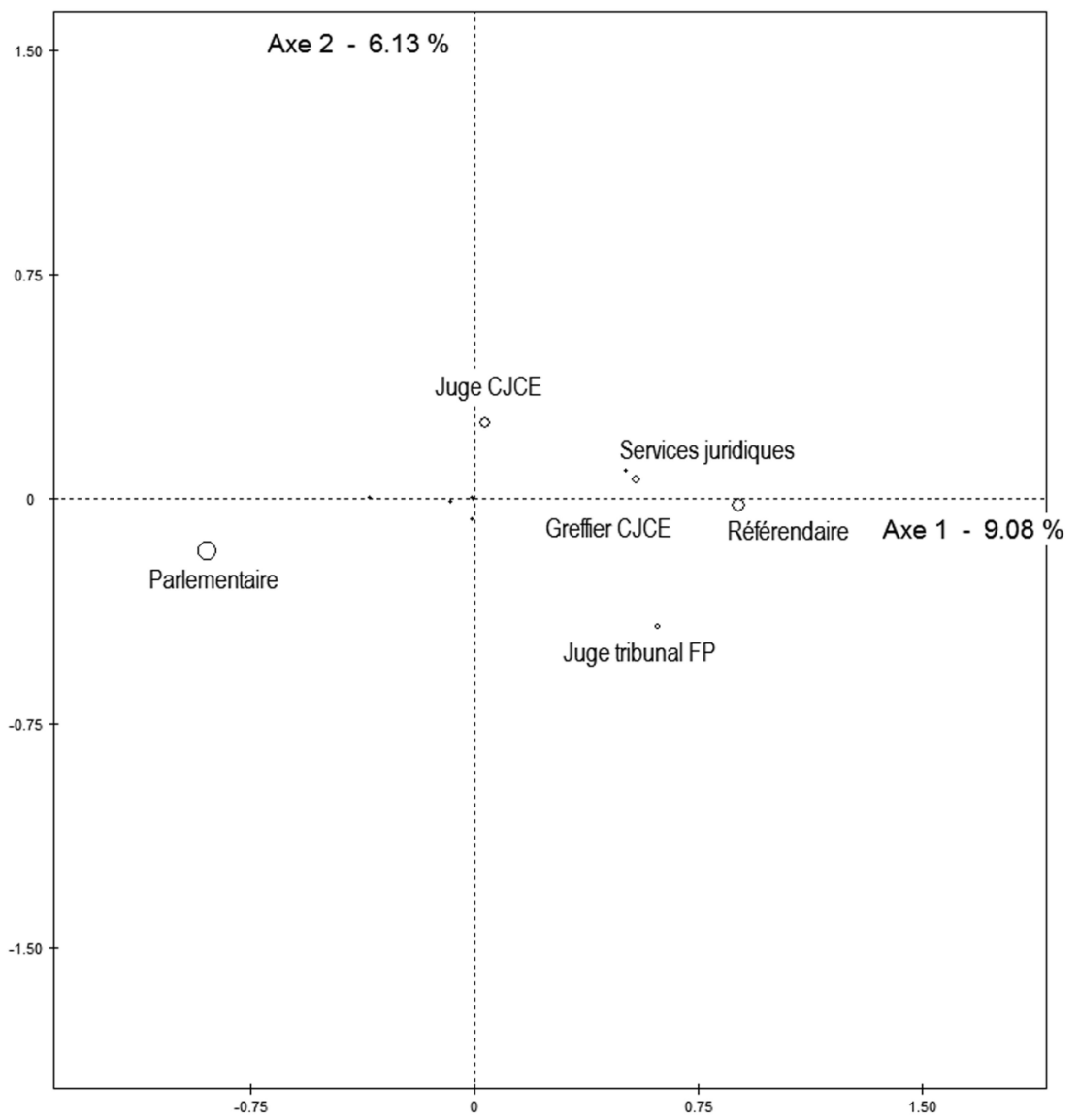

Graphique 7 : Projection des différentes " populations » en éléments supplémentaires dans le plan 1-2 
L'analyse menée permet ainsi de mieux spécifier les propriétés relationnelles des sous-populations identifiées comme des composantes d'un champ juridique européen, en ayant défini ces propriétés sur la base des différences systématiques de trajectoires individuelles (ce qui conduit à ne pas oublier qu'il ne s'agit que des moyennes de propriétés individuelles qui peuvent être dispersées). Bien évidemment, la construction du champ européen est nécessairement partielle, et ne fournit qu'une représentation heuristique d'un espace transnational multidimensionnel, permettant de dépasser des représentations figées et juxtaposées des différents groupes.

\section{Exemple 3. Esquisse d'un espace de la gouvernance économique européenne}

On a procédé pour analyser la structure de cet espace européen à une ACM spécifique. On a retenu comme variables actives de l'analyse (entre parenthèses le nombre de modalités actives) les quatorze variables suivantes :

1/ Propriétés socio-démographiques (deux variables, cinq modalités) : sexe (deux modalités), âge au moment de la publication de la notice (trois modalités : moins de 45 ans / 45-60 ans / 60 ans et plus);

2/ Trajectoire scolaire (huit variables, dix-neuf modalités) : lieu principal de formation (trois modalités), lieu du dernier diplôme (trois modalités), niveau d'études (trois modalités), Économie (deux modalités), Droit (deux modalités), Gestion (deux modalités), Sciences Po-Administration publique (deux modalités), Sciences (deux modalités);

3/ Carrière (quatre variables, neuf modalités) : expérience antérieure dans une administration (deux modalités), a été membre de cabinet (trois modalités), mobilité sectorielle (deux modalités), a occupé une position horizontale (deux modalités).

\begin{tabular}{|c|c|c|c|c|c|}
\hline Numéro & Valeur propre & $\begin{array}{c}\text { Taux brut } \\
\text { de variance }\end{array}$ & $\begin{array}{c}\text { Taux brut } \\
\text { de variance } \\
\text { cumulé }\end{array}$ & $\begin{array}{c}\text { Taux } \\
\text { de variance } \\
\text { modifié }\end{array}$ & $\begin{array}{c}\text { Taux modifié } \\
\text { cumulé }\end{array}$ \\
\hline 1 & 0,176 & 12,9 & 12,9 & $\mathbf{5 5 , 6} \%$ & $\mathbf{5 5 , 6} \%$ \\
\hline 2 & 0,105 & 7,7 & 20,7 & $\mathbf{1 1 , 1} \%$ & $\mathbf{6 6 , 6} \%$ \\
\hline 3 & 0,102 & 7,5 & 28,2 & $9,8 \%$ & $76,4 \%$ \\
\hline 5 & 0,095 & 7,0 & 35,1 & $7,4 \%$ & $83,8 \%$ \\
\hline 5 & 0,089 & 6,5 & 41,7 & $5,6 \%$ & $89,4 \%$ \\
\hline
\end{tabular}

Tableau 3 : Valeurs propres, taux de variance bruts et taux modifiés des cinq premiers axes

On retient ici trois axes, compte tenu de la décroissance des valeurs propres (mais l'on n'étudiera ici que les deux premiers). 


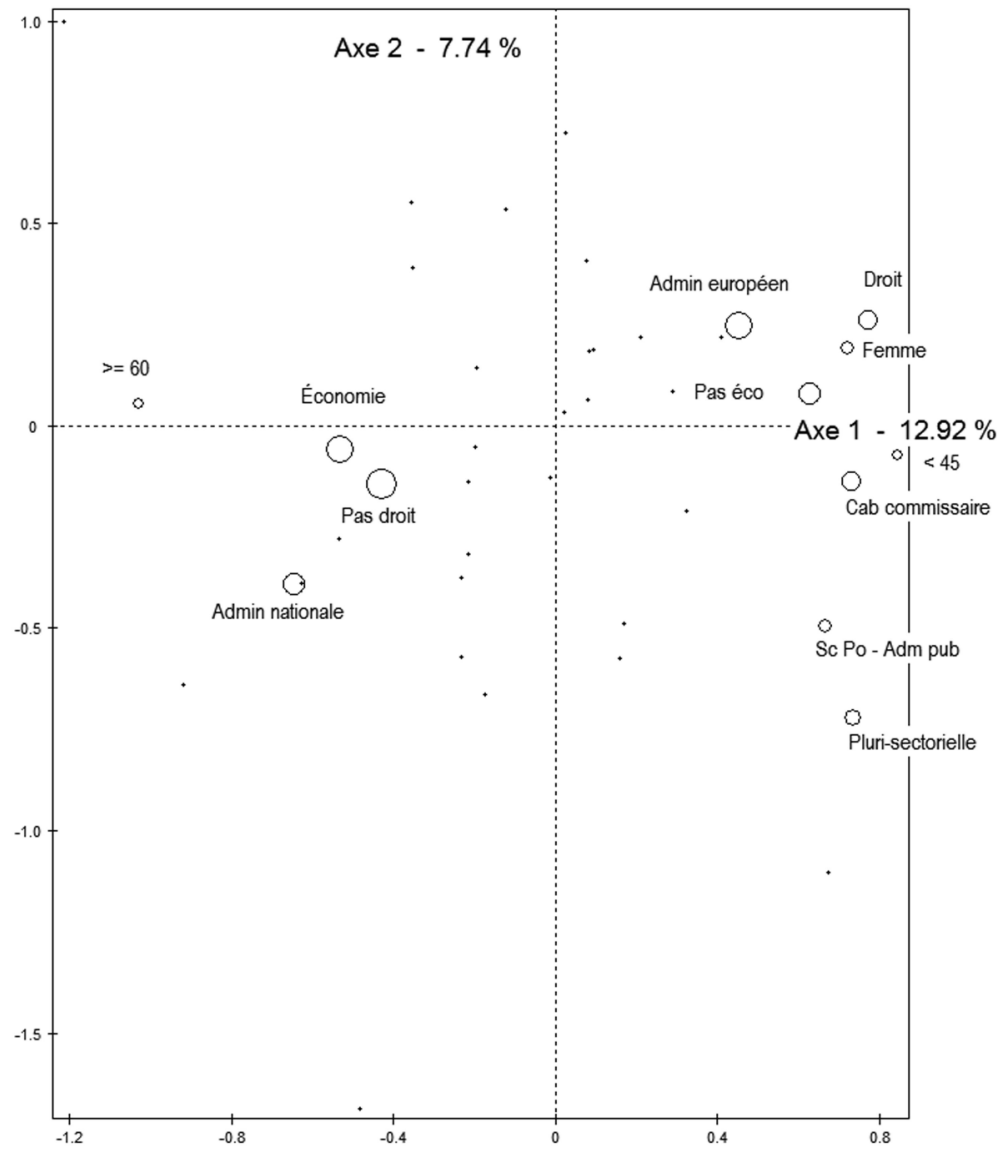

Graphique 8 : Modalités (30\%) les plus contributives sur I'axe 1

L'axe 1 oppose à gauche (valeurs négatives) : économie, pas de droit, administration nationale, et à droite : cabinet de commissaire, droit, pas d'économie, administration européenne, mobilité pluri-sectorielle, etc.

On a ici assez clairement une opposition entre le capital lié à la science économique, avec une composante étasunienne, mais lié à l'administration nationale, au capital juridique, qui apparait comme plus spécifique des institutions européennes ( Bruxelles») proprement dites. On trouve donc ici une différenciation selon le niveau d'européanisation du capital détenu, qui est inversement proportionnelle à la détention de capital en science économique.

L'axe 2, avant tout déterminé par les questions relatives à la carrière, oppose (en haut) l'absence de mobilité horizontale, de passage par un cabinet, de mobilité sectorielle, l'administration européenne, le passage par une grande université étasunienne, au (en bas) passage par un cabinet de dirigeant, la 


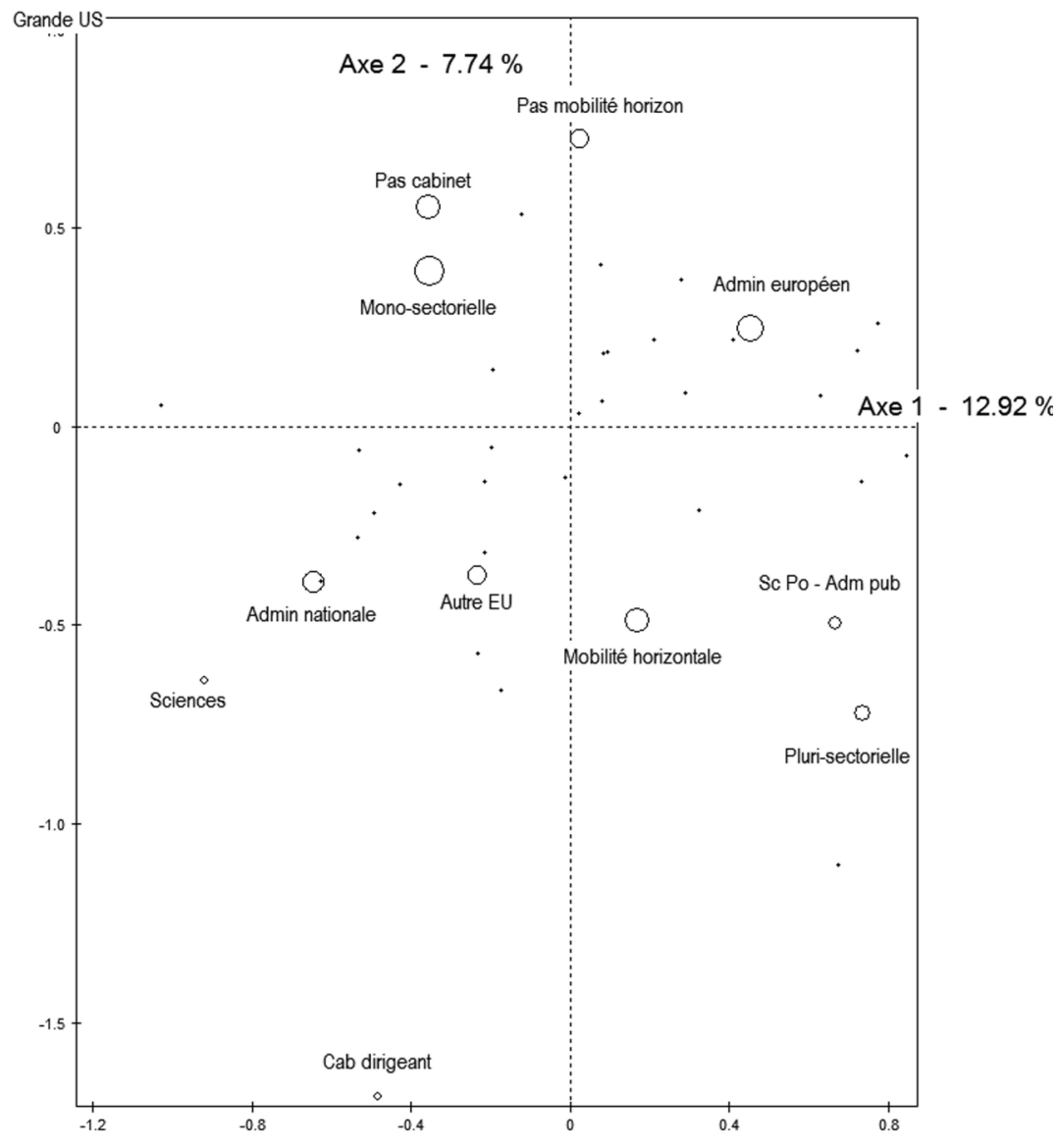

Graphique 9 : Modalités (30\%) les plus contributives sur l'axe 2

mobilité horizontale et plurisectorielle, l'administration nationale et les sciences, les sciences politiques.

On a ici un axe de surface positionnelle (elle augmente lorsque l'on descend le long de l'axe), de capital politique (avec le passage par un cabinet de dirigeant politique), y compris national (passage par l'administration nationale), avec la détention de diplômes en science politique.

On observe bien une relation étroite entre les capitaux détenus et les positions institutionnelles occupées dans le sous-champ de la gouvernance économique, projetées en éléments supplémentaires. Cela confirme qu'il est quelque peu vain d'opposer les ressources détenues par un individu et les effets de sa position officielle (institutionnelle), dans la mesure où les deux éléments interagissent fortement, les caractéristiques «incorporées »dans une trajectoire spécifique contribuant à déterminer le sens, l'efficacité et l'autorité de la posi- 
tion, et la position étant en elle-même une ressource fondamentale de l'action concrète de l'individu.

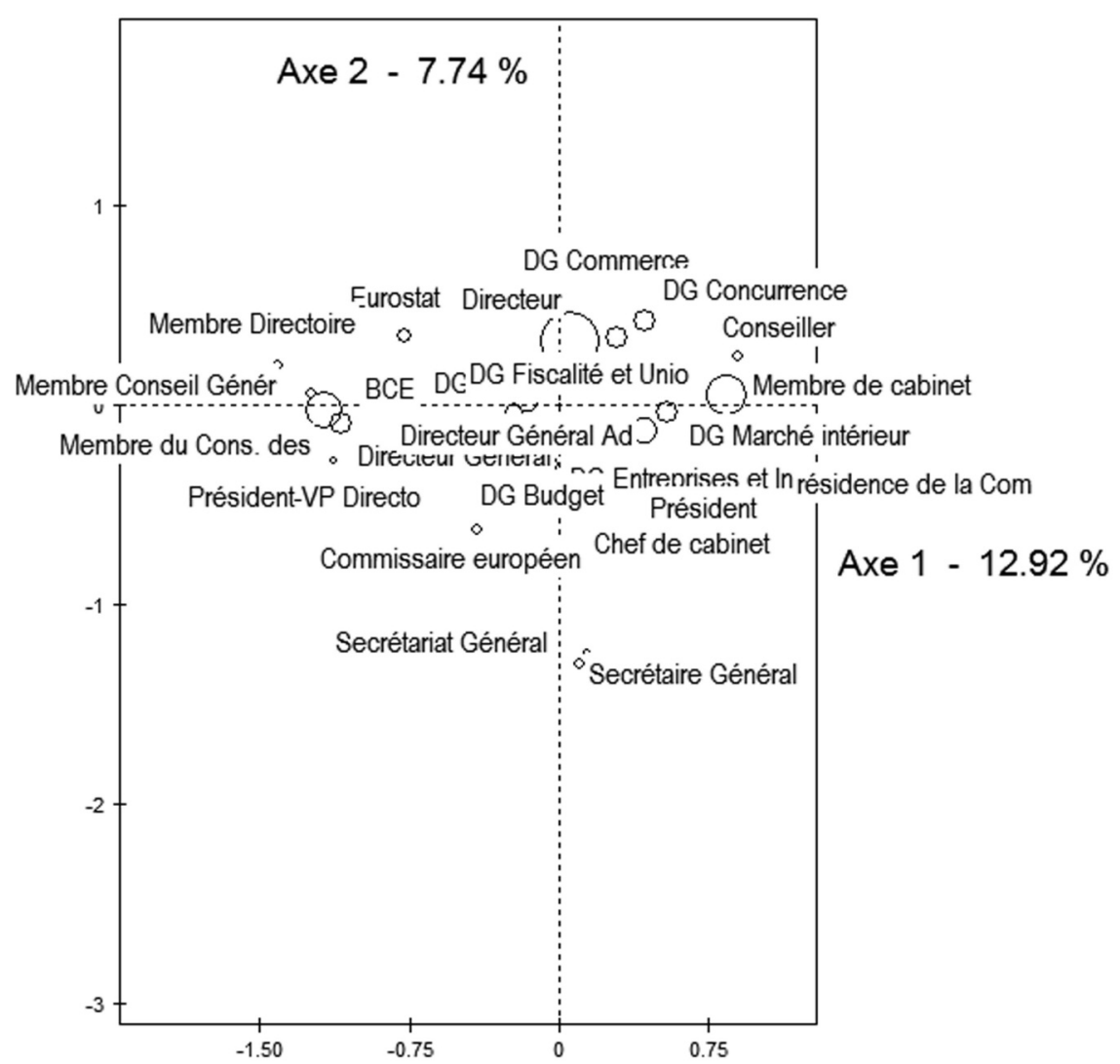

Graphique 10 : Positions et institutions (éléments supplémentaires) dans le plan 1-2

L'axe 1 oppose les positions au sein de la BCE à gauche des positions de chefs et membres de cabinet à droite, les positions les plus spécifiques à gauche des positions les plus « européennes » à droite. On observe donc déjà, à ce niveau, une forte relation entre les capitaux des acteurs et les institutions, qui isole l'univers particulier de la BCE («Francfort») à gauche.

L'axe 2 oppose clairement des positions à dominante politique (en bas), telles que secrétaire général de l'UE et commissaire, à des positions administratives « pures » (en haut), telles que celles de directeur.

S'agissant des institutions, l'espace est clairement divisé (axe 1) entre un pôle lié à la science économique, incarné par la BCE, mais aussi par Eurostat, et, dans une moindre mesure, la Directorate General for Economic and Financial Affairs (DG EcFin), d'une part, et les directions les plus « juri- 
diques » au sein du sous-champ de la gouvernance économique, comme la DG «marché intérieur » d'autre part.

L'axe 2 isole (en bas) le secrétariat général du conseil de l'UE et, dans une moindre mesure, la présidence de la Commission, lieux où l'on trouve donc le plus de surface, de circulation et de capital politique.

L'espace ainsi construit permet, à nouveau, de situer tout individu de la population étudiée dans une perspective relationnelle : on voit que les institutions se distribuent dans cet espace multidimensionnel, dont le point commun est d'être constitué par les acteurs occupant des positions de gouvernance économique.

Le nuage des individus fait apparaittre les positions occupées non plus par des institutions mais par les acteurs eux-mêmes, ce qui permet de surmonter l'opposition factice entre le niveau des acteurs individuels et celui des acteurs institutionnels.

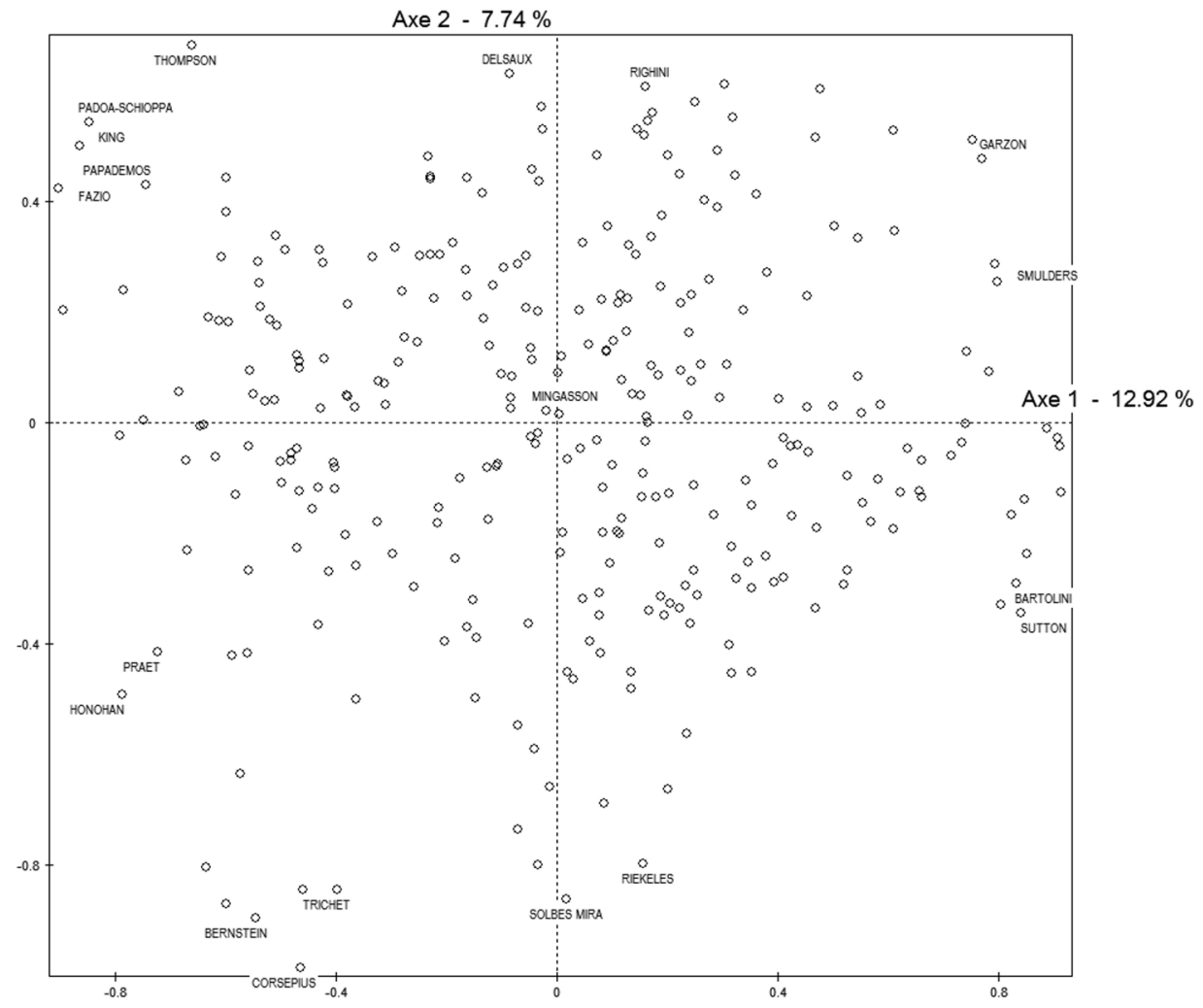

Graphique 11 : Nuage des individus dans le plan 1-2 (avec noms d'individus typiques de différents pôles) 
L'étude des champs transnationaux menée à l'aide des méthodes d'analyse géométrique des données constitue une pratique de longue durée, assez ingrate dans la lourde phase de constitution de l'information, mais aux nombreuses potentialités heuristiques en ce qui concerne l'exploration d'espaces sociaux complexes, en complément étroit avec l'utilisation de méthodes ou démarches plus qualitatives, centrées sur l'analyse et l'interprétation de parcours biographiques.

Elle suppose des investissements relativement lourds (en matière de budget, de temps, etc.). Elle constitue cependant un engagement individuel et collectif dont les retombées scientifiques sont peut-être encore sous-estimées, notamment parce qu'elle stimule des dynamiques de recherche collaboratives de plus en plus nécessaires s'agissant d'espaces transnationaux et qu'elle ouvre aussi des perspectives en matière de comparaison des structures macro ou méso-sociales, tout en reposant sur des observations établies à l'échelle des individus.

Plusieurs échelles et plusieurs niveaux d'information sont possibles, allant de la « petite base » individuelle à la grande base collective, de la base comme produit de la thèse ou de grands programmes de recherche transnationaux, ensuite partiellement exploitée par un ou plusieurs chercheurs.

Cette perspective implique de penser de manière relationnelle, c'est-à-dire en termes d'espace social, les objets étudiés, ce qui en fait un outil pour l'analyse qualitative autant que quantitative ${ }^{22}$. Elle peut notamment permettre la constitution systématique de biographies structurales ou relationnelles, qui évitent certaines des limites de la biographie classique, et en premier lieu « l'illusion biographique». Elle fournit enfin un moyen de tester la portée empirique des concepts théoriques et d'affiner la théorie sur des questions spécifiques posées aux données.

La portée de cette perspective de recherche est particulièrement claire dans le cas de l'étude d'espaces transnationaux, pour diverses raisons qui ont été évoquées dans cet article : la tentation de réifier les entités collectives est d'autant plus forte que l'on analyse, à l'échelle transnationale, des espaces relativement abstraits, d'où l'intérêt de représenter relationnellement, et de façon

22. Pour une élaboration et une discussion de cette perspective, voir : Bigo D., The field of the EU internal security agencies, Paris, L'Harmattan, 2007 ; Dezalay Y., " De la défense de l'environnement au développement durable. L'émergence d'un champ d'expertise des politiques européennes ", Actes de la recherche en sciences sociales, n 166-167, 2007, pp. 67-79; Fligstein N., McAdam D., A Theory of Fields, Oxford, Oxford University Press, 2013 ; Vauchez A., "The force of a weak field: law and lawyiers in the government of the European Union », International Political Sociology, vol. 2, n 2, 2008, pp. 128-144. 
synthétique, des espaces complexes, fortement multidimensionnels, qui échappent assez largement à l'intuition des acteurs, en y situant les acteurs individuels.

En définissant ces espaces sur la base de caractéristiques biographiques simples (et suffisamment comparables pour faire sens à l'échelle de ces espaces), on « re-peuple » ainsi les analyses sociologiques sur la base des trajectoires concrètes d'acteurs transnationaux, ce qui permet de consolider les analyses et hypothèses nourries par l'étude des biographies des acteurs individuels et par l'étude des « carrières » des institutions et des organisations. 